\title{
Recursions of Symmetry Orbits and Reduction without Reduction ${ }^{\star}$
}

\author{
Andrei A. MALYKH ${ }^{\dagger}$ and Mikhail B. SHEFTEL $\ddagger$ \\ $\dagger$ Department of Numerical Modelling, Russian State Hydrometeorlogical University, \\ Malookhtinsky pr. 98, 195196 St. Petersburg, Russia \\ E-mail: andrei-malykh@mail.ru \\ ¥Department of Physics, Boğaziçi University 34342 Bebek, Istanbul, Turkey \\ E-mail: mikhail.sheftel@boun.edu.tr
}

Received January 29, 2011, in final form April 25, 2011; Published online April 29, 2011

doi:10.3842/SIGMA.2011.043

\begin{abstract}
We consider a four-dimensional PDE possessing partner symmetries mainly on the example of complex Monge-Ampère equation (CMA). We use simultaneously two pairs of symmetries related by a recursion relation, which are mutually complex conjugate for CMA. For both pairs of partner symmetries, using Lie equations, we introduce explicitly group parameters as additional variables, replacing symmetry characteristics and their complex conjugates by derivatives of the unknown with respect to group parameters. We study the resulting system of six equations in the eight-dimensional space, that includes CMA, four equations of the recursion between partner symmetries and one integrability condition of this system. We use point symmetries of this extended system for performing its symmetry reduction with respect to group parameters that facilitates solving the extended system. This procedure does not imply a reduction in the number of physical variables and hence we end up with orbits of non-invariant solutions of CMA, generated by one partner symmetry, not used in the reduction. These solutions are determined by six linear equations with constant coefficients in the five-dimensional space which are obtained by a three-dimensional Legendre transformation of the reduced extended system. We present algebraic and exponential examples of such solutions that govern Legendre-transformed Ricci-flat Kähler metrics with no Killing vectors. A similar procedure is briefly outlined for Husain equation.
\end{abstract}

Key words: Monge-Ampère equation; partner symmetries; symmetry reduction; noninvariant solutions; anti-self-dual gravity; Ricci-flat metric

2010 Mathematics Subject Classification: 35Q75; 83C15

\section{Introduction}

In our recent papers $[1,2,3,4,5]$ we demonstrated how to use partner symmetries for obtaining non-invariant solutions of four-dimensional PDEs of Monge-Ampère type, which determine Ricci-flat metrics with no Killing vectors in the anti-self-dual gravity. Here we present another method of solving this problem by introducing explicitly group parameters of two pairs of partner symmetries (mutually complex conjugate for real equations like CMA) as additional variables in the system of four equations that determine recursions between the partner symmetries. For this purpose, we use Lie equations of partner symmetries for replacing their symmetry characteristics with derivatives of the unknown with respect to group parameters. Such an approach can be applied to any non-linear PDE of the Monge-Ampère type which admits a symmetry condition to be of a divergence form. The resulting system of five PDEs, that also includes the studied equation of the Monge-Ampère type, has one non-trivial integrability condition, which means

\footnotetext{
*This paper is a contribution to the Special Issue "Symmetry, Separation, Super-integrability and Special Functions $\left(\mathrm{S}^{4}\right)$ ". The full collection is available at http://www.emis.de/journals/SIGMA/S4.html
} 
that both pairs of partner symmetries commute with each other, so that we end up with six PDEs for one unknown in the eight-dimensional space of independent variables.

The problem of finding a solution of this extended system becomes much easier if we use three point symmetries of this system for performing its symmetry reduction with respect to a combination of variables involving group parameters. (One symmetry is not used for the reduction and so we still keep the dependence of solutions on one group parameter.) This does not mean a reduction in the number of "physical variables" because only group parameters disappear from the set of independent variables. Hence we still obtain a noninvariant solution that depends on the four physical variables and one remaining group parameter. Performing Legendre transformation in two physical variables and one group parameter we arrive at a system of six linear equations with constant coefficients for one unknown with five independent variables. One can easily construct many explicit solutions of this system which govern the Legendre-transformed heavenly metric. To illustrate this program, we consider CMA as a main example together with a brief outline of a similar procedure for the Husain equation. For the elliptic CMA we construct explicitly algebraic and exponential solutions.

We emphasize that our task here is not to obtain solutions of the original CMA equation, which would require application of the inverse Legendre transformation in two variables to our solutions, but rather to construct Ricci-flat vacuum metrics (solutions of Einstein equations with either Euclidean or neutral signature) with no Killing vectors (continuous symmetries). For this purpose, we apply the inverse one-dimensional Legendre transformation with respect to the group parameter to noninvariant solutions of the linear system which results in solutions of the Legendre-transformed CMA that determine metrics with the above properties. The general form of these metrics is obtained by a two-dimensional Legendre transformation of the Kähler metric with respect to a couple of complex conjugate physical variables.

In Section 2, we derive an over-determined system of six equations in eight-dimensional space with one unknown and a set of eight independent variables containing four symmetry group parameters. This system does not generate further independent second-order integrability conditions and it determines a class of solutions of the elliptic and hyperbolic CMA.

In Section 3, we list all point symmetries of this extended system and perform a symmetry reduction with respect to two group parameters. We calculate all point symmetries of the resulting system of six equations in six-dimensional space and use one of the symmetries for further reduction of this system with respect to one more group parameter, with one group parameter still remaining as the fifth independent variable.

In Section 4 we show how by applying Legendre transformation in three variables, including the group parameter, one arrives at a system of linear partial differential equations with constant coefficients for one unknown in a five-dimensional space, which does not generate further independent second-order differential constraints. Many explicit exact solutions of this system can easily be obtained.

In Section 5, we obtain the Legendre transform of the Kähler heavenly metric, using Legendre transformation with respect to two "physical" variables only. One should be careful to perform correctly three-dimensional Legendre transformation of the metric involving a transformation with respect to the group parameter. Otherwise, the differential of the group parameter appears in the transformed metric which results in the metric not being Ricci-flat anymore. Therefore, in the next section we have to apply the inverse one-dimensional Legendre transformation in the group parameter to solutions of the linear system in order to obtain the potential $v$, that governs the Legendre-transformed metric.

In Section 6, we present examples of exact algebraic and exponential non-invariant solutions of our equations which determine Ricci-flat metrics not admitting Killing vectors.

In Section 7, we derive an overdetermined system of six PDEs with one unknown and a set of eight variables containing four symmetry group parameters, which determines a class of 
solutions of the Husain equation. This system includes one second-order integrability condition of the other five equations and it has no further second-order integrability conditions.

\section{Basic equations}

We are interested in non-invariant solutions of the complex Monge-Ampère equation (CMA)

$$
u_{1 \overline{1}} u_{2 \overline{2}}-u_{1 \overline{2}} u_{2 \overline{1}}=\varepsilon, \quad \varepsilon= \pm 1,
$$

which is closely related to the first heavenly equation of Plebañski [6]. Symmetry condition, that determines symmetry characteristics $[7] \varphi$ of $(2.1)$,

$$
u_{1 \overline{1}} \varphi_{2 \overline{2}}+u_{2 \overline{2}} \varphi_{1 \overline{1}}-u_{1 \overline{2}} \varphi_{2 \overline{1}}-u_{2 \overline{1}} \varphi_{1 \overline{2}}=0
$$

can be set in the total divergence form

$$
\left(u_{1 \overline{1}} \varphi_{2}-u_{2 \overline{1}} \varphi_{1}\right)_{\overline{2}}-\left(u_{1 \overline{2}} \varphi_{2}-u_{2 \overline{2}} \varphi_{1}\right)_{\overline{1}}=0 .
$$

This suggests local existence of potential $\psi$ defined by the equations

$$
\psi_{\overline{1}}=u_{1 \overline{1}} \varphi_{2}-u_{2 \overline{1}} \varphi_{1}, \quad \psi_{\overline{2}}=u_{1 \overline{2}} \varphi_{2}-u_{2 \overline{2}} \varphi_{1}
$$

together with the complex conjugate equations

$$
\bar{\psi}_{1}=u_{1 \overline{1}} \bar{\varphi}_{\overline{2}}-u_{1 \overline{2}} \bar{\varphi}_{\overline{1}}, \quad \bar{\psi}_{2}=u_{2 \overline{1}} \bar{\varphi}_{\overline{2}}-u_{2 \overline{2}} \bar{\varphi}_{\overline{1}} .
$$

A straightforward check shows that the potential $\psi$ also satisfies symmetry condition (2.2), so that $\psi$ is also a symmetry if $\varphi$ is a symmetry and hence the relations (2.3) and (2.4) are recursion relations for symmetries ("partner symmetries"). Transformation (2.3) is algebraically invertible since its determinant is equal to $\varepsilon$ due to (2.1). Inverse transformation has the form

$$
\varphi_{1}=\varepsilon\left(u_{1 \overline{2}} \psi_{\overline{1}}-u_{1 \overline{1}} \psi_{\overline{2}}\right), \quad \varphi_{2}=\varepsilon\left(u_{2 \overline{2}} \psi_{\overline{1}}-u_{2 \overline{1}} \psi_{\overline{2}}\right)
$$

together with its complex conjugate

$$
\bar{\varphi}_{\overline{1}}=\varepsilon\left(u_{2 \overline{1}} \bar{\psi}_{1}-u_{1 \overline{1}} \bar{\psi}_{2}\right), \quad \bar{\varphi}_{\overline{2}}=\varepsilon\left(u_{2 \overline{2}} \bar{\psi}_{1}-u_{1 \overline{2}} \bar{\psi}_{2}\right) .
$$

For symmetries with characteristics $\varphi, \bar{\varphi}, \psi$ and $\bar{\psi}$, Lie equations read

$$
\varphi=u_{\tau}, \quad \bar{\varphi}=u_{\bar{\tau}}, \quad \psi=u_{\sigma}, \quad \bar{\psi}=u_{\bar{\sigma}},
$$

where $\tau, \sigma$ together with their complex conjugates $\bar{\tau}, \bar{\sigma}$ are group parameters. Simultaneous inclusion of several group parameters as additional independent variables implies the commutativity conditions for corresponding symmetries in the form

$$
\varphi_{\bar{\tau}}=\bar{\varphi}_{\tau}, \quad \psi_{\bar{\sigma}}=\bar{\psi}_{\sigma}, \quad \varphi_{\sigma}=\psi_{\tau}, \quad \varphi_{\bar{\sigma}}=\bar{\psi}_{\tau}
$$

and complex conjugates to the last two equations.

We now use (2.7) to replace symmetry characteristics by derivatives of $u$ with respect to group parameters in equations (2.3), (2.5) and their complex conjugates (2.4), (2.6) with the result

$$
\begin{array}{ll}
u_{\sigma \overline{1}}=u_{1 \overline{1}} u_{\tau 2}-u_{2 \overline{1}} u_{\tau 1}, & u_{\sigma \overline{2}}=u_{1 \overline{2}} u_{\tau 2}-u_{2 \overline{2}} u_{\tau 1}, \\
u_{\tau 1}=\varepsilon\left(u_{1 \overline{2}} u_{\sigma \overline{1}}-u_{1 \overline{1}} u_{\sigma \overline{2}}\right), & u_{\tau 2}=\varepsilon\left(u_{2 \overline{2}} u_{\sigma \overline{1}}-u_{2 \overline{1}} u_{\sigma \overline{2}}\right),
\end{array}
$$


and the complex conjugate equations

$$
\begin{aligned}
& u_{\bar{\sigma} 1}=u_{1 \overline{1}} u_{\bar{\tau} \overline{2}}-u_{\overline{2} 1} u_{\bar{\tau} \overline{1}}, \quad u_{\bar{\sigma} 2}=u_{\overline{1} 2} u_{\bar{\tau} \overline{2}}-u_{2 \overline{2}} u_{\bar{\tau} \overline{1}}, \\
& u_{\bar{\tau} \overline{1}}=\varepsilon\left(u_{\overline{1} 2} u_{\bar{\sigma} 1}-u_{1 \overline{1}} u_{\bar{\sigma} 2}\right), \quad u_{\bar{\tau} \overline{2}}=\varepsilon\left(u_{2 \overline{2}} u_{\bar{\sigma} 1}-u_{\overline{2} 1} u_{\bar{\sigma} 2}\right) .
\end{aligned}
$$

We note that four equations (2.9) and (2.11) are algebraic consequences of other equations (2.8), (2.10) and CMA. We note also that CMA itself follows as an algebraic consequence from equations (2.8) and (2.10). We choose equations (2.8), (2.10) and the first equation in (2.11) (since we regard CMA as algebraically dependent equation) as a set of five algebraically independent equations.

One can easily check that cross-differentiations of these equations with respect to group parameters yield only identities, e.g. $\left(u_{\sigma \overline{1}}\right)_{\bar{\tau}}=\left(u_{\bar{\tau} \overline{1}}\right)_{\sigma}$ implies

$$
\left(u_{1 \overline{1}} u_{\tau 2}-u_{2 \overline{1}} u_{\tau 1}\right)_{\bar{\tau}}=\varepsilon\left(u_{2 \overline{1}} u_{1 \bar{\sigma}}-u_{1 \overline{1}} u_{2 \bar{\sigma}}\right)_{\sigma}
$$

which is identically satisfied due to existing equations.

To study further differential integrability conditions of our system, we set the first equations in (2.8) and (2.11) in the form

$$
\left(u_{1 \overline{1}} u_{2}\right)_{\tau}=\left(u_{\sigma}+u_{2} u_{\tau 1}\right)_{\overline{1}}, \quad\left(u_{1 \overline{1}} u_{2}\right)_{\bar{\sigma}}=\left(u_{2} u_{\bar{\sigma} 1}-\varepsilon u_{\bar{\tau}}\right)_{\overline{1}} .
$$

Equations (2.12) constitute an active system since they have a second order nontrivial integrability condition obtained by cross-differentiation of these equations with respect to $\bar{\sigma}$ and $\tau$ and further integration with respect to $\bar{z}^{1}$

$$
\varepsilon u_{\tau \bar{\tau}}+u_{\sigma \bar{\sigma}}+u_{\bar{\sigma} 2} u_{\tau 1}-u_{\bar{\sigma} 1} u_{\tau 2}=0
$$

where the "constant" of integration can be eliminated by a redefinition of $u$. To make this equation self-conjugate, we multiply it with an overall factor $u_{1 \overline{1}}$ and then eliminate $u_{1 \overline{1}} u_{\bar{\sigma} 2}$ and

$u_{1 \overline{1}} u_{\tau 2}$ in the last two terms of (2.12) using first equations in (2.11) and (2.8), respectively, with the final form of the integrability condition

$$
u_{1 \overline{1}}\left(\varepsilon u_{\tau \bar{\tau}}+u_{\sigma \bar{\sigma}}\right)-\varepsilon u_{\tau 1} u_{\bar{\tau} \overline{1}}-u_{\sigma \overline{1}} u_{\bar{\sigma} 1}=0 .
$$

In a similar way we obtain another form of the integrability condition

$$
u_{2 \overline{2}}\left(\varepsilon u_{\tau \bar{\tau}}+u_{\sigma \bar{\sigma}}\right)-\varepsilon u_{\tau 2} u_{\bar{\tau} \overline{2}}-u_{\sigma \overline{2}} u_{\bar{\sigma} 2}=0 .
$$

We can choose (2.8), (2.10), (2.13) and first equation in (2.11) for the set of algebraically independent equations. All other equations, including CMA, are linearly dependent on the chosen equations. One could also check that there are no further independent second-order differential integrability conditions of our system of six equations.

\section{Symmetry reduction of extended system}

From now on we set $\varepsilon=+1$ and thereby concentrate on the elliptic CMA as an example. We list the generators of all point symmetries of the extended system of six equations CMA, (2.8), (2.10) and (2.13) (first equation in (2.11) also admits these symmetries as an obvious consequence)

$$
\begin{aligned}
& X_{1}=\partial_{\tau}, \quad \bar{X}_{1}=\partial_{\bar{\tau}}, \quad X_{2}=\partial_{\sigma}, \quad \bar{X}_{2}=\partial_{\bar{\sigma}}, \quad X_{3}=\tau \partial_{\tau}+\sigma \partial_{\sigma} \\
& \bar{X}_{3}=\bar{\tau} \partial_{\bar{\tau}}+\bar{\sigma} \partial_{\bar{\sigma}}, \quad X_{4}=z^{2} \partial_{2}-\bar{z}^{2} \partial_{\overline{2}}+\bar{\tau} \partial_{\bar{\tau}}-\tau \partial_{\tau}+\sigma \partial_{\sigma}-\bar{\sigma} \partial_{\bar{\sigma}} \\
& X_{5}=\tau \partial_{\bar{\sigma}}-\sigma \partial_{\bar{\tau}}, \quad \bar{X}_{5}=\bar{\tau} \partial_{\sigma}-\bar{\sigma} \partial_{\tau}, \quad X_{6}=z^{2} \partial_{2}+\bar{z}^{2} \partial_{\overline{2}}+u \partial_{u}
\end{aligned}
$$




$$
\begin{aligned}
& X_{a}=a\left(z^{1}, z^{2}, \bar{\tau}, \sigma\right) \partial_{u}, \quad X_{\bar{a}}=\bar{a}\left(\bar{z}^{1}, \bar{z}^{2}, \tau, \bar{\sigma}\right) \partial_{u} \\
& X_{c}=c_{z^{1}} \partial_{2}-c_{z^{2}} \partial_{1}+\left(\tau c_{\sigma}-\bar{\sigma} c_{\bar{\tau}}\right) \partial_{u}, \\
& X_{\bar{c}}=\bar{c}_{\bar{z}^{1}} \partial_{\overline{2}}-\bar{c}_{\bar{z}^{2}} \partial_{\overline{1}}+\left(\bar{\tau} \bar{c}_{\bar{\sigma}}-\sigma \bar{c}_{\tau}\right) \partial_{u}, \quad X_{f}=f(\tau, \sigma, \bar{\tau}, \bar{\sigma}) \partial_{u},
\end{aligned}
$$

where $a, \bar{a}, c=c\left(z^{1}, z^{2}, \bar{\tau}, \sigma\right), \bar{c}=\bar{c}\left(\bar{z}^{1}, \bar{z}^{2}, \tau, \bar{\sigma}\right)$ are arbitrary functions and $f(\tau, \sigma, \bar{\tau}, \bar{\sigma})$ satisfies the equation $f_{\tau \bar{\tau}}+f_{\sigma \bar{\sigma}}=0$. We note that obvious translational symmetry generators $\partial_{1}, \partial_{\overline{1}}, \partial_{2}$ and $\partial_{\overline{2}}$ are particular cases of the generators $X_{c}$ and $X_{\bar{c}}$.

We also note that if we perform the symmetry reduction with respect to the two symmetries $X_{2}-\gamma X_{1}$ and $\bar{X}_{2}-\bar{\gamma} \bar{X}_{1}$ in (3.1) simultaneously, then solutions satisfy the additional constraints $u_{\sigma}=\gamma u_{\tau}$ and $u_{\bar{\sigma}}=\bar{\gamma} u_{\bar{\tau}}$. In this case our equation (2.13) becomes

$$
(1+\gamma \bar{\gamma}) u_{1 \overline{1}} u_{\tau \bar{\tau}}-u_{1 \tau} u_{\overline{1} \bar{\tau}}-\gamma \bar{\gamma} u_{1 \bar{\tau}} u_{\overline{1} \tau}=0
$$

that is equivalent to the general heavenly equation of B. Doubrov and E. Ferapontov [8], which depends essentially on a single parameter.

We specify two symmetries from (3.1) for a symmetry reduction of the extended system

$$
X_{I}=\partial_{\tau}-\lambda \partial_{1}, \quad \bar{X}_{I}=\partial_{\bar{\tau}}-\bar{\lambda} \partial_{\overline{1}},
$$

where $\lambda$ is a constant parameter with $|\lambda|=1$, so that $\bar{\lambda}=\lambda^{-1}$. Solutions of CMA invariant with respect to symmetries (3.3) are determined by the conditions

$$
u_{\tau}=\lambda u_{1}, \quad u_{\bar{\tau}}=\lambda^{-1} u_{\overline{1}}
$$

Using (3.4), we eliminate $u_{\tau}$ and $u_{\bar{\tau}}$ in all the equations (2.8), (2.10), first equation in (2.11) and (2.13) to obtain

$$
\begin{aligned}
& u_{\sigma \overline{1}}=\lambda\left(u_{1 \overline{1}} u_{12}-u_{2 \overline{1}} u_{11}\right), \quad u_{\sigma \overline{2}}=\lambda\left(u_{1 \overline{2}} u_{12}-u_{2 \overline{2}} u_{11}\right), \\
& u_{\bar{\sigma} 1}=\lambda^{-1}\left(u_{1 \overline{1}} u_{\overline{1} \overline{2}}-u_{\overline{2} 1} u_{\overline{1} \overline{1}}\right), \quad u_{\bar{\sigma} 2}=\lambda^{-1}\left(u_{\overline{1} 2} u_{\overline{1} \overline{2}}-u_{2 \overline{2}} u_{\overline{1} \overline{1}}\right), \\
& u_{\overline{1} \overline{1}}=\lambda\left(u_{\overline{1} 2} u_{\bar{\sigma} 1}-u_{1 \overline{1}} u_{\bar{\sigma} 2}\right), \\
& u_{1 \overline{1}} u_{\sigma \bar{\sigma}}-u_{1 \bar{\sigma}} u_{\overline{1} \sigma}=u_{11} u_{\overline{1} \overline{1}}-u_{1 \overline{1}}^{2} .
\end{aligned}
$$

We note that equation (3.8) can be obtained by the Legendre transformation

$$
v=u-z^{1} u_{1}-\bar{z}^{1} u_{\overline{1}}, \quad p=-u_{1}, \quad \bar{p}=-u_{\overline{1}}
$$

of the CMA in new variables

$$
v_{p \bar{p}} v_{\sigma \bar{\sigma}}-v_{p \bar{\sigma}} v_{\sigma \bar{p}}=1
$$

All point symmetry generators of the system of equations CMA, (3.5), (3.6) and (3.8) (and the equation in (3.7) as a consequence) are listed below

$$
\begin{aligned}
& X_{1}=z^{1} \partial_{1}-\bar{z}^{1} \partial_{\overline{1}}-2\left(z^{2} \partial_{2}-\bar{z}^{2} \partial_{\overline{2}}\right), \quad X_{2}=z^{2} \partial_{2}+\bar{z}^{2} \partial_{\overline{2}}+u \partial_{u}, \\
& X_{3}=a(\sigma) \partial_{2}+\frac{1}{2 \lambda}\left(z^{1}\right)^{2} a^{\prime}(\sigma) \partial_{u}, \quad X_{4}=b(\bar{\sigma}) \partial_{\overline{2}}+\frac{\lambda}{2}\left(\bar{z}^{1}\right)^{2} b^{\prime}(\bar{\sigma}) \partial_{u}, \\
& X_{5}=c^{\prime}(\sigma)\left(z^{1} \partial_{1}-z^{2} \partial_{2}\right)+c(\sigma) \partial_{\sigma}-\frac{1}{2 \lambda}\left(z^{1}\right)^{2} z^{2} c^{\prime \prime}(\sigma) \partial_{u}, \\
& X_{6}=d^{\prime}(\bar{\sigma})\left(\bar{z}^{1} \partial_{\overline{1}}-\bar{z}^{2} \partial_{\overline{2}}\right)+d(\bar{\sigma}) \partial_{\bar{\sigma}}-\frac{\lambda}{2}\left(\bar{z}^{1}\right)^{2} \bar{z}^{2} d^{\prime \prime}(\bar{\sigma}) \partial_{u}, \\
& X_{7}=-\lambda f_{z^{2}}\left(z^{2}, \sigma\right) \partial_{1}+z^{1} f_{\sigma}\left(z^{2}, \sigma\right) \partial_{u}, \quad X_{8}=-\frac{1}{\lambda} g_{\bar{z}^{2}}\left(\bar{z}^{2}, \bar{\sigma}\right) \partial_{\overline{1}}+\bar{z}^{1} g_{\bar{\sigma}}\left(\bar{z}^{2}, \bar{\sigma}\right) \partial_{u},
\end{aligned}
$$




$$
X_{9}=h\left(z^{2}, \sigma\right) \partial_{u}, \quad X_{10}=k\left(\bar{z}^{2}, \bar{\sigma}\right) \partial_{u}
$$

To simplify the problem of solving our equations, we make another symmetry reduction with respect to group parameters that does not imply a reduction in the original "physical" variables. For this purpose, we use the symmetry $X_{5}-i X_{6}=i\left(\partial_{\sigma}-\partial_{\bar{\sigma}}\right)$ from the list (3.9) taken at $c(\sigma)=1$ and $d(\bar{\sigma})=1$. The invariance condition for solutions is $u_{\sigma}=u_{\bar{\sigma}}=u_{\rho}$, where $\rho=\sigma+\bar{\sigma}$ is the invariant variable which replaces $\sigma$ and $\bar{\sigma}$ as an argument of $u$. Equations (3.5) and (3.6) become

$$
u_{\rho \overline{1}}=\lambda\left(u_{1 \overline{1}} u_{12}-u_{2 \overline{1}} u_{11}\right), \quad u_{\rho \overline{2}}=\lambda\left(u_{1 \overline{2}} u_{12}-u_{2 \overline{2}} u_{11}\right)
$$

together with their complex conjugates, while first equation in (2.11) becomes

$$
u_{\overline{1} \overline{1}}=\lambda\left(u_{\overline{1} 2} u_{\rho 1}-u_{1 \overline{1}} u_{\rho 2}\right)
$$

and equation (3.8) takes the form

$$
u_{1 \overline{1}} u_{\rho \rho}-u_{1 \rho} u_{\overline{1} \rho}=u_{11} u_{\overline{1} \overline{1}}-u_{1 \overline{1}}^{2} .
$$

\section{Linearizing Legendre transformation}

Equation (3.12) is linearized by the three-dimensional Legendre transformation

$$
u=w-p w_{p}-\bar{p} w_{\bar{p}}-r w_{r}, \quad z^{1}=-w_{p}, \quad \bar{z}^{1}=-w_{\bar{p}}, \quad \rho=-w_{r},
$$

where $w=w(p, \bar{p}, r, z, \bar{z})$ depends on the new variables $p, \bar{p}, r, z=z^{2}, \bar{z}=\bar{z}^{2}$. Equation (3.12) takes the form $\left(w_{p \bar{p}}+w_{r r}\right) / D=0$, where

$$
D \stackrel{\text { def }}{=}\left|\begin{array}{lll}
w_{p p} & w_{p \bar{p}} & w_{p r} \\
w_{\bar{p} p} & w_{\bar{p} \bar{p}} & w_{\bar{p} r} \\
w_{r p} & w_{r \bar{p}} & w_{r r}
\end{array}\right| \neq 0
$$

is the existence condition for Legendre transformation (4.1). Thus, equation (3.12) becomes a linear equation

$$
w_{p \bar{p}}+w_{r r}=0,
$$

while the first and second equations (3.10) and their complex conjugates take the form

$$
\begin{aligned}
& w_{p p} w_{\bar{p} r}-w_{p \bar{p}} w_{p r}-\lambda\left(w_{\bar{p} r} w_{z r}-w_{\bar{p} z} w_{r r}\right)=0, \\
& w_{p \bar{p}}\left(w_{p \bar{p}} w_{\bar{z} r}-w_{p \bar{z}} w_{\bar{p} r}\right)+\lambda w_{\bar{p} r}\left(w_{\bar{p} r} w_{z \bar{z}}-w_{\bar{p} z} w_{\bar{z} r}\right) \\
& \quad+w_{\bar{p} \bar{p}}\left[w_{p \bar{z}} w_{p r}-w_{p p} w_{\bar{z} r}+\lambda\left(w_{z r} w_{\bar{z} r}-w_{z \bar{z}} w_{r r}\right)\right] \\
& \quad-w_{\bar{p} \bar{z}}\left[w_{p \bar{p}} w_{p r}-w_{p p} w_{\bar{p} r}+\lambda\left(w_{\bar{p} r} w_{z r}-w_{\bar{p} z} w_{r r}\right)\right]=0,
\end{aligned}
$$

respectively, together with their complex conjugates and equation (3.11) reads

$$
w_{p p} w_{r r}-w_{p r}^{2}-\lambda\left(w_{p r} w_{\bar{p} z}-w_{p \bar{p}} w_{z r}\right)=0
$$

after canceling nonzero factor $D$ due to condition (4.2). Eliminating $w_{p \bar{p}}$ from (4.4) with the aid of (4.3), we obtain (4.4) in the form

$$
w_{\bar{p} r}\left(\lambda w_{z r}-w_{p p}\right)-w_{r r}\left(w_{p r}+\lambda w_{\bar{p} z}\right)=0 .
$$


Similarly, using (4.3) in (4.6), we obtain

$$
w_{r r}\left(\lambda w_{z r}-w_{p p}\right)+w_{p r}\left(w_{p r}+\lambda w_{\bar{p} z}\right)=0 .
$$

Determinant of the algebraic system of two latter equations is nonvanishing: $w_{r r}^{2}+w_{p r} w_{\bar{p} r} \neq 0$, since otherwise $w_{r r}=0$ and $w_{p r}=w_{\bar{p} r}=0$ separately which contradicts condition (4.2). Therefore, equations (4.7) and (4.8) become

$$
w_{p p}-\lambda w_{z r}=0, \quad w_{p r}+\lambda w_{\bar{p} z}=0 .
$$

Now we use linear equations (4.3), (4.9) and their complex conjugates in equation (4.5) to obtain

$$
\left(w_{p \bar{p}}+w_{z \bar{z}}\right)\left(w_{p \bar{p}}^{2}+w_{p \bar{z}} w_{\bar{p} z}\right)=0
$$

which obviously implies

$$
w_{p \bar{p}}+w_{z \bar{z}}=0,
$$

since otherwise $w_{p \bar{p}}^{2}+w_{p \bar{z}} w_{\bar{p} z}=0$ implies that $w_{p \bar{p}}=0$ and $w_{p \bar{z}}=w_{\bar{p} z}=0$, which means symmetry reduction with respect to physical variables that we wish to avoid. Thus, we end up with the six linear equations (4.3), (4.9), (4.10) and complex conjugates of the two equations (4.9). One can check that the complete system of six second-order linear equations does not generate further second order integrability conditions.

We emphasize that these six linear equations were obtained by symmetry reduction in the number of group parameters only, with one remaining Legendre transformed group parameter $r$. No reduction with respect to original "physical" variables has been performed, so that we are able to obtain noninvariant solutions of CMA by solving linear equations with constant coefficients. Noninvariant solutions of the general heavenly equation (3.2) can also be obtained by solving a linear system.

We note that Legendre transformation with respect to symmetry group parameters was also used by M. Dunajski and L.J. Mason for linearization of equations that determine invariant solutions of the hierarchy of the second heavenly equation of Plebañski [9].

\section{Transformation of Kähler metric}

Solutions $u=u\left(z^{1}, \bar{z}^{1}, z^{2}, \bar{z}^{2}\right)$ of the complex Monge-Ampère equation (2.1) govern the Kähler metric [6]

$$
d s^{2}=2 u_{1 \overline{1}} d z^{1} d \bar{z}^{1}+2 u_{1 \overline{2}} d z^{1} d \bar{z}^{2}+2 u_{2 \overline{1}} d z^{2} d \bar{z}^{1}+2 u_{2 \overline{2}} d z^{2} d \bar{z}^{2}
$$

which is an (anti-)self-dual Ricci-flat metric and hence it satisfies vacuum Einstein equations with Euclidean signature.

To obtain a correct transformation of the metric (5.1), we restrict transformation (4.1) to physical variables only, so that the Legendre transformation reads

$$
\begin{aligned}
& u=v-p v_{p}-\bar{p} v_{\bar{p}}, \quad z^{1}=-v_{p}, \quad \bar{z}^{1}=-v_{\bar{p}}, \quad z^{2}=z, \quad \bar{z}^{2}=\bar{z}, \\
& d z^{1}=-\left(v_{p p} d p+v_{p \bar{p}} d \bar{p}+v_{p z} d z+v_{p \bar{z}} d \bar{z}\right), \\
& d \bar{z}^{1}=-\left(v_{\bar{p} p} d p+v_{\bar{p} \bar{p}} d \bar{p}+v_{\bar{p} z} d z+v_{\bar{p} \bar{z}} d \bar{z}\right),
\end{aligned}
$$

where $v$ is related to $w$ in (4.1) by the one-dimensional Legendre transformation (5.4). Metric (5.1) after transformation (5.2) becomes

$$
\begin{aligned}
d s^{2}= & \frac{1}{\Delta}\left[v_{p p}\left(v_{\bar{p} p} d p+v_{\bar{p} z} d z\right)^{2}+v_{\bar{p} \bar{p}}\left(v_{p \bar{p}} d \bar{p}+v_{p \bar{z}} d \bar{z}\right)^{2}\right] \\
& +\frac{\Delta_{+}}{v_{p \bar{p}} \Delta}\left(v_{\bar{p} p} d p+v_{\bar{p} z} d z\right)\left(v_{p \bar{p}} d \bar{p}+v_{p \bar{z}} d \bar{z}\right)+\frac{\Delta}{v_{p \bar{p}}} d z d \bar{z},
\end{aligned}
$$

where $\Delta=v_{p p} v_{\bar{p} \bar{p}}-v_{p \bar{p}}^{2}, \Delta_{+}=v_{p p} v_{\bar{p} \bar{p}}+v_{p \bar{p}}^{2}$. 
We are able to construct many solutions $w=w(p, \bar{p}, z, \bar{z}, r)$ of the system of six linear equations with constant coefficients: (4.3), (4.10), (4.9) and complex conjugates of the two latter equations. In order to reconstruct corresponding solutions $v(p, \bar{p}, z, \bar{z}, \rho)$ that govern metric (5.3), we will use the one-dimensional Legendre transformation

$$
\rho=-w_{r}, \quad v=w-r w_{r}, \quad r=v_{\rho} .
$$

For given $w(p, \bar{p}, z, \bar{z}, r)$, equations (5.4) determine the function $v(p, \bar{p}, z, \bar{z}, \rho)$ in a parametric form where $r$ is the parameter. In examples of the next section, we show how to obtain an explicit form of the potential $v$ of the metric (5.3) by applying an inverse Legendre transformation with respect to the group parameter to a solution $w$ of the linear system.

\section{Examples of exact solutions determining Ricci-flat metrics with no Killing vectors}

Here we present some examples of non-invariant solutions of our equations that determine Ricciflat metrics of the form (5.3) that do not admit Killing vectors. For simplicity, we set $\lambda=1$ in equations (4.9).

One general type of solutions of linear equations (4.3), (4.9), (4.10) and complex conjugates of equations (4.9) has the form

$$
w=\int_{\alpha_{0}}^{\alpha_{1}} F\left(\alpha, \xi_{\alpha}+i \eta_{\alpha}\right) d \alpha+\sum_{j} F_{j}\left(\xi_{\alpha_{j}}+i \eta_{\alpha_{j}}\right)+\text { c.c. },
$$

where here and further c.c. means complex conjugate of the previous term(s), $\alpha$ is real,

$$
\begin{array}{rlrl}
\xi_{\alpha} & =p+\bar{p}+i \alpha(\bar{p}-p), & \eta_{\alpha}=\sqrt{\gamma}\left(r+\mu_{\alpha}\right), \\
\mu_{\alpha}=\frac{\alpha+i}{\alpha-i} z+\frac{\alpha-i}{\alpha+i} \bar{z}, & \gamma=\alpha^{2}+1,
\end{array}
$$

where bar means complex conjugate.

\subsection{Algebraic solution}

Here we restrict ourselves to the case of the discrete spectrum in (6.1) and the cubic solution of the form

$$
\begin{aligned}
w= & a\left(\xi_{\alpha}+i \eta_{\alpha}\right)^{3}+b\left(\xi_{\beta}+i \eta_{\beta}\right)^{3}+\text { c.c. } \\
= & A\left[\xi_{\alpha}^{3}-3 \gamma \xi_{\alpha}\left(r+\mu_{\alpha}\right)^{2}\right]+C\left[3 \xi_{\alpha}^{2}\left(r+\mu_{\alpha}\right)-\gamma\left(r+\mu_{\alpha}\right)^{3}\right] \\
& +B\left[\xi_{\beta}^{3}-3 \delta \xi_{\beta}\left(r+\mu_{\beta}\right)^{2}\right]+D\left[3 \xi_{\beta}^{2}\left(r+\mu_{\beta}\right)-\delta\left(r+\mu_{\beta}\right)^{3}\right],
\end{aligned}
$$

where $A, B, C, D$ are arbitrary real constants

$$
A=a+\bar{a}, \quad B=b+\bar{b}, \quad C=i \sqrt{\gamma}(a-\bar{a}), \quad D=i \sqrt{\delta}(b-\bar{b}),
$$

$\xi_{\beta}$ and $\mu_{\beta}$ are obtained from $\xi_{\alpha}$ and $\mu_{\alpha}$ in (6.2) by replacing $\alpha$ by $\beta$, setting $\delta=\beta^{2}+1$ and $\eta_{\beta}=\sqrt{\delta}\left(r+\mu_{\beta}\right)$. Solution of the form (6.3) is the simplest nontrivial algebraic solution in this class because a quadratic solution corresponds to constant metric coefficients in (5.3) and hence to a vanishing connection and curvature tensor. 
According to the Legendre transformation (5.4), $r$ is determined in terms of $\rho$ and "physical variables" by the equation $w_{r}+\rho=0$. With $w$ determined by (6.3), it takes the form of the quadratic equation for $r$

$$
k r^{2}+2 l r+m=0
$$

where

$$
\begin{aligned}
& k=c \gamma+D \delta, \quad l=\gamma\left(A \xi_{\alpha}+C \mu_{\alpha}\right)+\delta\left(B \xi_{\beta}+D \mu_{\beta}\right), \\
& m=\gamma\left(2 A \xi_{\alpha} \mu_{\alpha}+C \mu_{\alpha}^{2}\right)+\delta\left(2 B \xi_{\beta} \mu_{\beta}+D \mu_{\beta}^{2}\right)-C \xi_{\alpha}^{2}-D \xi_{\beta}^{2}-\frac{\rho}{3}
\end{aligned}
$$

with the solution

$$
r=\frac{-l \pm \sqrt{\Delta}}{k}, \quad \text { where } \quad \Delta=l^{2}-k m
$$

Next, we use (5.4) in the form $v=w+\rho r$ and replace $r$ by the expression (6.6) with the definitions (6.5) to obtain

$$
v=\frac{1}{k^{3}}\left(P_{3} \pm \sqrt{\Delta} P_{2}\right),
$$

where

$$
\begin{aligned}
P_{2}= & 6\left(A \gamma s_{\alpha} l_{\alpha}+B \delta s_{\beta} l_{\beta}\right)+C\left[3 s_{\alpha}^{2}-\gamma\left(\Delta+3 l_{\alpha}^{2}\right)\right]+D\left[3 s_{\beta}^{2}-\delta\left(\Delta+3 l_{\beta}^{2}\right)\right]+k^{2} \rho, \\
P_{3}= & A\left[s_{\alpha}^{3}-3 \gamma s_{\alpha}\left(\Delta+l_{\alpha}^{2}\right)\right]+B\left[s_{\beta}^{3}-3 \delta s_{\beta}\left(\Delta+l_{\beta}^{2}\right)\right] \\
& -3 C l_{\alpha}\left[s_{\alpha}^{2}-\gamma\left(\Delta+\frac{l_{\alpha}^{2}}{3}\right)\right]-3 D l_{\beta}\left[s_{\beta}^{2}-\delta\left(\Delta+\frac{l_{\beta}^{2}}{3}\right)\right]-k^{2} \rho l
\end{aligned}
$$

and $s_{\alpha}=k \xi_{\alpha}, s_{\beta}=k \xi_{\beta}, l_{\alpha}=l-k \mu_{\alpha}, l_{\beta}=l-k \mu_{\beta}$. Here $P_{2}$ and $P_{3}$ are polynomials in $p, \bar{p}, z$, $\bar{z}$ of degrees 2 and 3 respectively.

By construction, the expression (6.7) for $v$ satisfies the equation resulting after two dimensional Legendre transformation (5.2) of CMA equation (2.1) with $\varepsilon=+1$

$$
v_{p \bar{z}} v_{\bar{p} z}-v_{p \bar{p}} v_{z \bar{z}}+v_{p p} v_{\bar{p} \bar{p}}-v_{p \bar{p}}^{2}=0
$$

which governs the Legendre-transformed metric (5.3). Solutions (6.3) and (6.7) for $w$ and $v$ are obviously noninvariant, since there is no symmetry reduction in the number of independent variables in these formulas. Therefore, solution (6.7), being used in the metric (5.3), yields a Ricci-flat metric of Euclidean signature with no Killing vectors.

We can convert formula (6.7) for the solution to the polynomial form

$$
k^{6} v^{2}-2 k^{3} P_{3} v+P_{3}^{2}-\Delta P_{2}^{2}=0 .
$$

By the change of variables $v=V^{3}$ and $\rho=\sigma^{2}$, solution (6.9) becomes

$$
k^{6} V^{6}-2 k^{3} P_{3} V^{3}+P_{6}=0,
$$

where $P_{6}=P_{3}^{2}-\Delta P_{2}^{2}$ is a homogeneous polynomial of degree 6. Hence, our solution (6.10) is the set of roots of the homogeneous polynomial of degree 6 in the six variables $V, p, \bar{p}, z, \bar{z}, \sigma$. 
Remark 6.1. In our construction, we could skip the reality condition and replace the complex conjugate variables $\bar{z}^{1}, \bar{z}^{2}$ by two more independent complex variables $\tilde{z}^{1}$, $\tilde{z}^{2}$. Then CMA equation (2.1) is replaced by the first heavenly equation of Plebañski [6]

$$
u_{1 \tilde{1}} u_{2 \tilde{2}}-u_{1 \tilde{2}} u_{2 \tilde{1}}=1
$$

and its Legendre transform has the form (6.8) with $\bar{p}, \bar{z}$ replaced by $\tilde{p}, \tilde{z}$ respectively. Its algebraic solution is obtained from our real solution by the same replacements in formulas (6.2) with $u$ and $v$ considered now as holomorphic functions of complex arguments and arbitrary real constants replaced by complex ones. Such a complex solution, being the set of roots of the homogeneous polynomial of degree 6 in the six complex variables $V, p, \bar{p}, z, \bar{z}, \sigma$, determines a four-dimensional compact manifold in a five-dimensional projective space $\mathbb{C P}^{5}$ with the local coordinates $(V / \sigma, p / \sigma, \bar{p} / \sigma, z / \sigma, \bar{z} / \sigma)[10]$.

First example of an algebraic solution that governs anti-self-dual gravity was presented in our recent paper [11].

Ricci-flat metrics in Euclidean signature with no Killing vectors on a compact manifold are interesting because they satisfy necessary existence conditions for the famous gravitational instanton $K 3$ [12], whose explicit form is still unknown and presents an "outstanding open problem in Riemannian geometry and the theory of gravitational instantons" [13].

\subsection{Exponential solution}

Now we choose the exponential solution belonging to the discrete spectrum in (6.1)

$$
w=a e^{i\left(\xi_{\alpha}+i \eta_{\alpha}\right) / \sqrt{\gamma}}+\bar{a} e^{-i\left(\xi_{\alpha}-i \eta_{\alpha}\right) / \sqrt{\gamma}}+b e^{-i\left(\xi_{\beta}+i \eta_{\beta}\right) / \sqrt{\delta}}+\bar{b} e^{i\left(\xi_{\beta}-i \eta_{\beta}\right) / \sqrt{\delta}}
$$

with the same expressions for $\gamma, \delta$ and $\xi_{\alpha, \beta}, \eta_{\alpha, \beta}$ as before. After using these expressions in (6.11) together with the definitions of arbitrary real constants

$$
\begin{aligned}
& \cos \theta=\frac{1}{\sqrt{\gamma}}, \quad \sin \theta=\frac{\alpha}{\sqrt{\gamma}}, \quad \cos \phi=\frac{1}{\sqrt{\delta}}, \quad \sin \phi=\frac{\beta}{\sqrt{\delta}}, \\
& A=a+\bar{a}, \quad B=b+\bar{b}, \quad C=i(a-\bar{a}), \quad D=i(b-\bar{b})
\end{aligned}
$$

one obtains

$$
w=e^{-r} G(p, \bar{p}, z, \bar{z})+e^{r} H(p, \bar{p}, z, \bar{z}),
$$

where we have defined

$$
\begin{aligned}
G= & \exp [\cos (2 \theta)(z+\bar{z})+i \sin (2 \theta)(\bar{z}-z)] \\
& \times\{A \cos [\cos \theta(p+\bar{p})+i \sin \theta(\bar{p}-p)]+C \sin [\cos \theta(p+\bar{p})+i \sin \theta(\bar{p}-p)]\}, \\
H= & \exp [-\cos (2 \phi)(z+\bar{z})+i \sin (2 \phi)(z-\bar{z})] \\
& \times\{B \cos [\cos \phi(p+\bar{p})+i \sin \phi(\bar{p}-p)]-D \sin [\cos \phi(p+\bar{p})+i \sin \phi(\bar{p}-p)]\} .
\end{aligned}
$$

The equation $w_{r}+\rho=0$ following from (5.4) now becomes the quadratic equation for the exponential $e^{r}:\left(e^{r}\right)^{2} H+\rho e^{r}-G=0$ with the solution for $r$

$$
r=\ln \left\{\frac{-\rho \pm \sqrt{\rho^{2}+4 G H}}{2 H}\right\} .
$$

Using this expression for $r$ in equation (6.12) for $w$ and then in the equation $v=w+\rho r$ from (5.4), after elementary simplifications one obtains

$$
v= \pm \sqrt{\rho^{2}+4 G H}+\rho \ln \left\{\frac{-\rho \pm \sqrt{\rho^{2}+4 G H}}{2 H}\right\}
$$


with $G$ and $H$ defined by (6.13) and (6.14), respectively. By construction, the expression (6.15) satisfies the Legendre-transformed CMA equation (6.8) being a noninvariant solution of this equation, since there is obviously no symmetry reduction in the number of independent variables in this solution. Therefore, solution (6.15), being used in the metric (5.3), yields a Ricci-flat metric of Euclidean signature with no Killing vectors.

\section{Basic equations associated with the Husain equation}

Here we demonstrate that the same approach can be applied to the Husain equation [14]

$$
u_{t y} u_{x z}-u_{t z} u_{x y}+u_{t x}=0
$$

which can be brought to a standard form by a simple change of variables. We shall briefly revise the derivation of the basic equations from Section 2 in a form adapted to equation (7.1). If $\varphi$ is a symmetry characteristic of (7.1), the symmetry condition reads

$$
u_{x z} \varphi_{t y}+u_{t y} \varphi_{x z}-u_{x y} \varphi_{t z}-u_{t z} \varphi_{x y}+\varphi_{t x}=0 .
$$

Equation (7.2), taken in an explicitly divergence form, implies the existence of the potential $\psi$ connected with $\varphi$ by the relations

$$
\psi_{t}=\varphi_{t}+u_{t y} \varphi_{z}-u_{t z} \varphi_{y}, \quad \psi_{x}=u_{x y} \varphi_{z}-u_{x z} \varphi_{y}
$$

The potential $\psi$ is a symmetry characteristic of the Husain equation (7.1) as soon as $\varphi$ is also a symmetry characteristic, so that (7.3) determines a recursion relation for symmetries of (7.1). Using Lie equations (2.7), we replace symmetry characteristics $\psi, \varphi$ in (7.3) by derivatives of $u$ with respect to the group parameters $\alpha, \beta$ and $\gamma, \delta$, respectively:

$$
\begin{aligned}
& u_{\alpha t}=u_{\beta t}+u_{t y} u_{\beta z}-u_{t z} u_{\beta y}, \\
& u_{\alpha x}=u_{x y} u_{\beta z}-u_{x z} u_{\beta y}, \\
& u_{\gamma t}=u_{\delta t}+u_{t y} u_{\delta z}-u_{t z} u_{\delta y}, \\
& u_{\gamma x}=u_{x y} u_{\delta z}-u_{x z} u_{\delta y} .
\end{aligned}
$$

Equations (7.4) and (7.6), written in the form

$$
\begin{aligned}
& u_{\alpha t}=u_{\beta t}+\left(u_{t y} u_{z}\right)_{\beta}-\left(u_{z} u_{\beta y}\right)_{t}, \\
& u_{\gamma t}=u_{\delta t}+\left(u_{t y} u_{z}\right)_{\delta}-\left(u_{z} u_{\delta y}\right)_{t},
\end{aligned}
$$

imply the compatibility condition obtained by cross differentiating (7.8) and (7.9) with respect to $\delta$ and $\beta$, respectively, and integrating the result with respect to $t$

$$
u_{\alpha \delta}-u_{\beta \gamma}+u_{\beta y} u_{\delta z}-u_{\beta z} u_{\delta y}=0
$$

Here the "constant" of integration with respect to $t$ has been eliminated by a redefinition of $u$. Thus, we again have a system of six equations: (7.1), (7.4)-(7.7) and the integrability condition (7.10).

Applying the same strategy as in Section 3, we can perform symmetry reductions of this extended system with respect to group parameters, which do not imply reductions in the number of original physical variables, and apply an appropriate Legendre transform to obtain a system of easily solvable linear equations. 


\section{Conclusion}

We have shown that noninvariant solutions of the complex Monge-Ampère equation can be obtained in principle by solving an overdetermined system of six linear partial differential equations with constant coefficients for one unknown depending on five independent variables, that include a symmetry group parameter. We considered in detail the complex Monge-Ampère equation together with recursion relations for partner symmetries. Using Lie equations, we introduced explicitly four symmetry group parameters as additional independent variables, which enabled us to derive one integrability condition of the considered system. In this way, we acquired four additional independent variables, so that we could make symmetry reductions by eliminating three group parameters which facilitated solution process of our equations but in no way meant the reduction in the number of original "physical" variables. Using Legendre transformation in three variables, including the remaining group parameter, we arrived at linear partial differential equations with constant coefficients which determine symmetry group orbits of noninvariant solutions of the Legendre-transformed CMA. These equations can be easily solved and we have shown how to use these solutions for reconstructing explicitly the potential $v$ that governs the Legendre transform of the Kähler metric. We present algebraic and exponential examples of such noninvariant solutions which determine Ricci-flat metrics with the Euclidean signature not admitting any Killing vectors. We emphasize that our goal here was to obtain gravitational metrics with the above properties for which it was sufficient to obtain solutions of the Legendre-transformed CMA equation that yield solutions of CMA in a parametric form. To obtain an explicit form of the corresponding solutions of the original CMA, we have to apply an inverse two-dimensional Legendre transformation to our solutions for the potential $v$. We note that we could consider the first heavenly equation of Plebañski instead of the CMA with our solutions still being valid if we consider the variables $\bar{z}^{1}, \bar{z}^{2}$ not as complex conjugates to $z^{1}, z^{2}$ but rather as additional independent complex variables. We also note that in the same framework we may choose for the reduction many other symmetries from our lists. Solutions obtained with these alternative choices will be published elsewhere. We have also briefly outlined a similar approach for the Husain equation.

\section{Acknowledgements}

The authors thank M. Dunajski for an interesting discussion about the problem of performing the Legendre transformation of a metric with respect to a symmetry group parameter. The research of MBS was supported in part by the research grant from Bogazici University Scientific Research Fund, research project No. 07B301. AAM is grateful to Feza Gursey Institute for the support of his stay at Istanbul where this work was finalized.

\section{References}

[1] Sheftel M.B., Malykh A.A., On classification of second-order PDEs possessing partner symmetries, J. Phys. A: Math. Theor. 42 (2009), 395202, 20 pages, arXiv:0904.2909.

[2] Malykh A.A., Nutku Y., Sheftel M.B., Partner symmetries of the complex Monge-Ampère equation yield hyper-Kähler metrics without continuous symmetries, J. Phys. A: Math. Gen. 36 (2003), 10023-10037, math-ph/0305037.

[3] Malykh A.A., Nutku Y., Sheftel M.B., Partner symmetries and non-invariant solutions of four-dimensional heavenly equations, J. Phys. A: Math. Gen. 37 (2004), 7527-7545, math-ph/0403020.

[4] Malykh A.A., Nutku Y., Sheftel M.B., Lift of noninvariant solutions of heavenly equations from three to four dimensions and new ultra-hyperbolic metrics, J. Phys. A: Math. Theor. 40 (2007), 9371-9386, arXiv:0704.3335. 
[5] Sheftel M.B., Malykh A.A., Lift of invariant to non-invariant solutions of complex Monge-Ampère equations, J. Nonlinear Math. Phys. 15 (2008), suppl. 3, 375-385, arXiv:0802.1463.

[6] Plebañski J.F., Some solutions of complex Einstein equations, J. Math. Phys. 16 (1975), 2395-2402.

[7] Olver P.J., Applications of Lie groups to differential equations, Graduate Texts in Mathematics, Vol. 107, Springer-Verlag, New York, 1986.

[8] Doubrov B., Ferapontov E.V., On the integrability of symplectic Momge-Ampère equations, J. Geom. Phys. 60 (2010), 1604-1616, arXiv:0910.3407.

[9] Dunajski M., Mason L.J., Twistor theory of hyper-Kähler metrics with hidden symmetries, J. Math. Phys. 44 (2003), 3430-3454, math.DG/0301171.

[10] Griffiths P., Harris J., Principles of algebraic geometry, Wiley \& Sons, Inc., New York, 1994.

[11] Malykh A.A., Sheftel M.B., General heavenly equation governs anti-self-dual gravity, J. Phys. A: Math. Theor. 44 (2011), 155201, 11 pages, arXiv:1011.2479.

[12] Atiyah M.F., Hitchin N.J., Singer I.M., Self-duality in four-dimensional Riemannian geometry, Proc. Roy. Soc. London Ser. A $\mathbf{3 6 2}$ (1978), 425-461.

[13] Dunajski M., Solitons, instantons, and twistors, Oxford Graduate Texts in Mathematics, Vol. 19, Oxford University Press, Oxford, 2010.

[14] Husain V., Self-dual gravity as a two dimensional theory and conservation laws, Classical Quantum Gravity 11 (1994), 927-937, gr-qc/9310003. 